\title{
Two negative randomized controlled trials in lupus: now what?
}

\section{Andreea Coca and Jennifer H Anolik*}

\author{
Address: University of Rochester School of Medicine, Box 695, 601 Elmwood Avenue, Rochester, NY 14642, USA \\ *Corresponding author: Jennifer H Anolik (jennifer_anolik@urmc.rochester.edu) \\ FI000 Medicine Reports 2009, I:28 (doi: 10.3410/MI-28) \\ The electronic version of this article is the complete one and can be found at: http://www.FI000.com/Reports/Medicine/content/I/28
}

\begin{abstract}
Recently, two large randomized controlled trials of distinct biologic therapies in systemic lupus erythematosus, B-cell depletion with rituximab and co-stimulatory blockade with CTLA4Ig (abatacept), failed to meet primary endpoints. Given the great need for new treatments in lupus, these results were met with disappointment and have left the rheumatology and immunology community searching for an explanation. Are these experimental agents ineffective in lupus or are there trial design issues or other considerations? In this commentary, we discuss our perspective on these results within the context of current understanding of the pathophysiology of lupus and the mechanism of action of biologic therapies.
\end{abstract}

\section{Introduction and context}

Systemic lupus erythematosus (SLE) is a complex autoimmune disease with considerable heterogeneity, characterized by dysregulation of the immune system and production of hallmark autoantibodies. Because this disease continues to be associated with significant morbidity and a three- to fivefold increased mortality compared to the general population, there is great interest in the development of new and targeted treatment approaches [1]. Current treatments are generally broadly immunosuppressive and thus fraught with complications and sometimes lack of efficacy. However, major obstacles in finding efficacious therapies for SLE include the challenges of clinical trial design given the low prevalence of disease, great clinical heterogeneity, relapsing-remitting course, and lack of well-established endpoints [2-5]. These challenges have contributed to the fact that there have been no new drugs approved for the treatment of SLE in over 50 years.

Despite these difficulties, there is reason for optimism. A great deal of effort toward improving lupus clinical trial methodology has come from OMERACT (Outcome Measures in Rheumatology) [6], the US Food and Drug Administration (FDA) clinical trial guidelines [7], the Systemic Lupus International Collaborating Clinics conference, and EULAR (European League Against Rheumatism) [5]. Moreover, our understanding about the pathogenesis of SLE has grown substantially in the past decade, leading to an explosion of promising biologic therapies.

Although multiple immunologic abnormalities are undoubtedly important for the development and clinical expression of SLE, a large body of evidence strongly points to the $\mathrm{B}$ cell as a central player in the pathogenesis of this disease [8]. The disease is characterized not only by defects in B-cell development, homeostasis, signaling, and tolerance but also by abnormalities in cytokines and chemokines either produced by B cells or with important B-cell influences [8]. B cells may participate in the immune dysregulation of SLE at multiple levels; serving as the precursors of antibody-secreting cells, taking up and presenting autoantigens to $\mathrm{T}$ cells, helping to regulate and organize inflammatory responses through cytokine and chemokine secretion (such as interleukin10 , interleukin-6, interferon- $\gamma$, and lymphotoxin- $\alpha$ ), and regulating other immune cells. The importance of these latter functions has been clearly demonstrated in murine SLE, where B cells have been found to be critical to the development of disease even when they are unable to secrete autoantibodies [9]. 
Given the large body of evidence implicating abnormalities in the B-cell compartment in SLE, a rational approach to treatment involves B-cell depletion. FDAapproved for lymphoma and rheumatoid arthritis refractory to anti-tumor necrosis factor (TNF), B-cell depletion with the anti-CD20 monoclonal antibody rituximab has also shown benefit in open-label studies and case series (often for severe, refractory cases) for many other autoimmune diseases. As for SLE, our initial phase I/II study [10] and other similar open-label trials have provided strong support for this therapy, which has become a useful tool for refractory SLE with either renal, central nervous system, or hematological involvement [11-16]. Given the growing familiarity amongst rheumatologists with using this therapy in rheumatoid arthritis, there is expanding off-label use in other rheumatic diseases including SLE.

Rituximab is by no means the only promising biologic therapy under investigation in SLE. As an alternative to selective B-cell depletion, there has been great interest in targeting co-stimulatory signaling pathways as well as antagonizing cytokines that have prominent $\mathrm{B}$-cell effects. Belimumab is a fully human monoclonal antibody that neutralizes soluble B-cell activating factor of the TNF family (BAFF), a cytokine important for B-cell survival and plasma cell differentiation. Mice overexpressing BAFF develop an SLE-like phenotype [17]. Moreover, lupus-prone mice have elevated levels of circulating BAFF, and administration of soluble BAFF receptors ameliorates disease progression and improves survival [18].

Blockade of co-stimulatory interactions between B7 on antigen-presenting cells (including B cells) and CD28 on T cells can also prevent activation of B cells in SLE. A fusion protein of the extracellular domain of cytotoxic T-lymphocyte antigen (CTLA) and immunoglobulin constant regions, CTLA4Ig, binds to B7 at a much higher affinity than CD28, thus blocking CD28-mediated co-stimulation and activation of T cells. CTLA4Ig (abatacept) is FDA approved for the treatment for rheumatoid arthritis [19] and, thus, similar to rituximab, is theoretically available for off-label use in SLE. In murine SLE, CTLA4Ig has demonstrated remarkable efficacy, improving both renal disease and survival [20].

\section{Recent advances}

Randomized controlled trials evaluating either rituximab or abatacept in the treatment of SLE have not been available until the recently reported studies at the 2008 American College of Rheumatology meeting. Merrill et al. [21,22] reported the EXPLORER trial, a randomized, double-blind, placebo-controlled trial comparing rituximab to placebo in a 2:1 randomization in 257 active non-renal lupus patients. Inclusion criteria required at least one organ with British Isles Lupus Assessment Group (BILAG) A (severe disease activity) or at least two organs with BILAG B (moderate disease activity) at study entry while taking immune suppressive treatment. Two doses of 1,000 mg rituximab given 2 weeks apart were administered at baseline and again at 6 months. All patients continued their baseline treatments and received a 10 week course of high dose steroids up to 0.5 to $1.0 \mathrm{mg} / \mathrm{kg}$ depending on baseline steroids and the severity of their disease, with taper over an additional 8 weeks. The proportion of subjects achieving a partial clinical response (PCR) or major clinical response (MCR) at 52 weeks, with a primary endpoint as defined in Table 1, was no different in the treatment group (24.9\% PCR including MCR) versus the placebo group $(27.3 \%)$. There were also no differences between the groups for any of the pre-defined, mostly BILAG-based, secondary endpoints. Both groups had significant and sustained decreases in global BILAG and Systemic Lupus Erythematosus Disease Activity Index (SLEDAI) scores over time. In a pre-specified subgroup analysis of black and Hispanic subjects, who overall had lower placebo responses (15.7\% PCR including MCR), there was a statistically significant difference with the addition of rituximab (33.8\% PCR including MCR). Additionally, the subgroup of patients receiving methotrexate began to diverge at the end of the study, with a statistically significant increase in the placebo group disease activity and sustained response in the rituximab treated group. Finally, there was a suggestion of serological efficacy given the improvement in anti-DNA titers and complement levels in both groups, but with significant separation over time favoring rituximab. Overall, we conclude that the EXPLORER trial did not demonstrate clinical efficacy of rituximab in SLE since it did not meet any of the primary or secondary endpoints. However, the outcome suggests that most SLE patients with non-renal disease respond well to high dose steroids, and other immunosuppressive agents appear to be effective in maintaining a response, making it difficult to demonstrate a benefit of drug with this study design.

Merrill et al. also reported on an efficacy study of abatacept (CTLA4Ig) in reducing flares in non-renal SLE patients with active polyarthritis, serositis, or discoid lesions. This double-blind, placebo-controlled phase II trial randomized 180 subjects 2:1 to abatacept $(10 \mathrm{mg} / \mathrm{kg}$ every other week for three doses, then monthly) or placebo for 1 year. Prednisone at $30 \mathrm{mg}$ per day was given for 1 month and then tapered per protocol. The primary endpoint was the proportion of patients with a 
Table I. Recent SLE randomized, controlled trials of biologics

\begin{tabular}{lllll}
\hline Reference & Size & Study drug & Endpoint & Select findings \\
\hline Merrill et al. [2I] & 257 & Rituximab & $\begin{array}{l}\text { BILAG MCR/PCR* } \\
\text { at } 52 \text { weeks }\end{array}$ & $\begin{array}{l}\text { No significant difference in primary and secondary endpoints; subgroup analysis } \\
\text { suggests benefit in black and Hispanic subjects }\end{array}$ \\
Merrill et al. [22] & 180 & Abatacept & $\begin{array}{l}\text { BILAG flare by } \\
\text { I year }\end{array}$ & $\begin{array}{l}\text { No significant difference in primary and secondary endpoints; post hoc subgroup } \\
\text { analysis suggests higher fraction of treated subjects on low dose prednisone without } \\
\text { flare during final } 2 \text { months of study }\end{array}$ \\
Chatham et al. [23] & 449 & Belimumab & $\begin{array}{l}\text { SELENA-SLEDAl } \\
\text { at } 52 \text { weeks }\end{array}$ & $\begin{array}{l}\text { No significant difference in primary and secondary endpoints; post hoc subgroup } \\
\text { analysis suggests benefit in seropositive subjects as measured by novel SLE responder } \\
\text { index }\end{array}$
\end{tabular}

*MCR = all BILAG Cs or better in every organ system at 24 weeks with maintenance of this response until week $52 ;$ PCR = all BILAG Cs or better at week 24 and maintenance for 16 weeks or at most I BILAG B at week 24 and maintenance until week 52. BILAG, British Isles Lupus Assessment Group; MCR, major clinical response; PCR, partial clinical response; SELENA, Safety of Estrogen in Lupus Erythematosus National Assessment; SLE, systemic lupus erythematosus; SLEDAI, SLE Disease Activity Index.

new SLE flare (BILAG A or B) after the start of steroid taper over 1 year. There was no difference in the flare rates between the treatment group $(79.7 \%)$ and placebo group $(82.5 \%)$ or any of the secondary BILAG endpoints. In post hoc analyses, the question of whether BILAG B flare might be too stringent an endpoint to distinguish between placebo and active treatment was examined. An increased trend towards efficacy was found in the polyarthritis subgroup (95 patients) when only BILAG A flares were counted $(36.5 \%$ in abatacept-treated patients versus $62.5 \%$ in the placebo group). When physician assessment was used to determine flare there was a difference in overall flare endpoint $(63.6 \%$ in the abatacept group versus $82.3 \%$ in the placebo group). In addition, the proportion of patients who were on less than $7.5 \mathrm{mg}$ prednisone and also rated to have no flare during months 10-12 was significantly higher in the abatacept group (42.4 versus $28.1 \%$ in the placebo group). As with the prior trial, we conclude that this study did not demonstrate efficacy of abatacept in nonrenal SLE since the primary and secondary endpoints were not met. However, the post hoc analyses suggest to us that the outcome measures used were suboptimal and also that there may be greater efficacy of abatacept in the polyarthritis subset of disease.

As a point of comparison, belimumab (fully human monoclonal antibody against BAFF) for SLE has also been evaluated in a double-blind, placebo-controlled trial. Long-term follow-up for this trial was also presented at the American College of Rheumatology 2008 meeting [23]. This study did not meet its original primary efficacy endpoint, which was the change in the Safety of Estrogen in Lupus Erythematosus National Assessment (SELENA)-SLEDAI score at week 52. However, $28 \%$ of patients were not serologically active [serologically active patients were defined as antinuclear antibody positive (+ANA) or anti-double-stranded DNA (dsDNA) positive and represented $72 \%(n=321)$ of study patients]. When only the serologically active subjects were analyzed post hoc, there was a statistically significant improvement in disease activity using a novel responder index [the SLE responder index (SRI)] based on a SELENA-SLEDAI improvement of four or more, no new BILAG $1 \mathrm{~A}$ or $2 \mathrm{~B}$ flares, and no worsening on the Physicians Global Assessment. As defined by the SRI, belimumab-treated seropositive subjects achieved a $46 \%$ response at week 52 versus $29 \%$ of placebo-treated subjects $(P<0.05)$. With open-label therapy, the SRI response increased to $55 \%$ by week 76 and persisted through 160 weeks. By week 52, 62\% of belimumabtreated seropositive subjects had SELENA-SLEDAI flares versus $74 \%$ of placebo-treated subjects ( $P$-values not significant). During long-term open treatment, the frequency of SELENA-SLEDAI flares declined to $7 \%$ at 3 years, suggestive of sustained improvement of disease activity with long-term therapy. Overall, we conclude that belimumab may have efficacy in non-renal SLE, particularly over long-term treatment, results that need to be confirmed in ongoing randomized studies with appropriately selected SLE patients and refined primary endpoints.

\section{Implications for clinical practice}

There are two broad explanations for the negative results of these two recently reported major trials: either the drugs are not effective or the trial design was suboptimal for demonstrating clinical efficacy. We do concede that animal models do not always accurately reflect what will happen in humans. Moreover, there have been notable examples of therapeutics that appeared effective in openlabel studies until well controlled studies provided contrary evidence [24]. Nevertheless, given the large body of pre-clinical and open-label data in refractory disease suggesting efficacy, we should consider whether trial design was optimal for demonstrating benefit. Several recent editorials have addressed the formidable challenges of trial design in SLE $[2,3,25]$. No drug has 
been approved for SLE in the past 50 years. Therefore, we do not have established methods for determining efficacy for new drugs, especially for non-renal SLE. The discussion that follows focuses on a number of key trial design issues within the context of the expected biological action of rituximab and abatacept.

\section{Timing of endpoints}

Steroids have a rapid onset of action but other agents may take time to become effective. Indeed, the results of the open-label extension of the belimumab trial suggest that endpoints longer than 1 year may be necessary to determine the full potential of biologic therapy. In EXPLORER, greater serological improvement (antidsDNA, C3, C4) was seen in patients treated with rituximab compared to placebo by the end of the trial. Although serological improvement was not associated with clinical improvement, a longer trial may have found benefit.

In the EXPLORER trial, this problem may have been compounded by re-treatment with rituximab at 6 months. One of the interesting findings from our pilot open-label trial of rituximab in SLE was the prolonged effect on the immune system with an expansion of transitional B-cells at reconstitution in patients who were good long-term responders [26]. We hypothesize that this B-cell subset may have immunoregulatory potential. If so, re-treatment with rituximab every 6 months would prevent the beneficial effects of an expanded transitional cell subset. This raises the important issue that both the timing and choice of endpoints need to be matched to the putative mechanism of action of the biologic agent. Even more, one might hypothesize that the treatment should be even more individualized, based on the degree of B-cell depletion. We have observed various degrees of residual memory B-cells in our rituximab-treated SLE patients, which we believe may adversely affect outcomes [27].

\section{Choice of endpoints}

None of the primary outcome measures used in any of the three discussed trials had been validated in clinical trials. Although a significant improvement in SLEDAI score of four points should provide good evidence of efficacy of an agent, this may not be a sensitive enough tool especially when measured against an effective 'placebo'. We believe the BILAG-based MCRs and PCRs defined in the EXPLORER study are also problematic. By these indexes, response needed to be achieved within 6 months and then maintained. However, based on the mechanism of action of rituximab in SLE, maximal response may not be achieved by 6 months. As previously discussed, we believe that depletion of $\mathrm{B}$ cells is responsible for only a fraction of the efficacy of
B-cell depletion therapy. Other putative critical mechanisms include the way the immune system reconstitutes (in which case long follow-up will be necessary beyond 1 year) and/or indirect effects of B-cell depletion on other arms of the immune system, like T cell subsets.

Secondary endpoints in EXPLORER (largely BILAG based), including the time to moderate or severe BILAG flare, and exploratory endpoints, including a decrease in global BILAG and SLEDAI score, were also not met. However, the abatacept study seems to suggest that outcome indicators that use BILAG flares as the measurement tool are also problematic, especially if a single BILAG B flare is counted. The novel SRI defined in the belimumab study post hoc analysis may provide a more sensitive and accurate measure of disease response. It notably combines the SLEDAI, the BILAG, and Physician Global Assessment, and has apparently been accepted by the FDA as an SLE disease assessment tool. Additional work is necessary in the lupus community, however, to decide upon and further validate appropriate composite outcome measures.

Another consideration is whether quantitative measures of disease activity in defined organ systems would provide more objective outcomes. Of note, ongoing trials of rituximab and abatacept in renal lupus have the dual advantage of hard outcomes in a more homogeneous population of SLE. On the other hand, outcome measures can only be part of the problem given the recent press release from Genentech that their study of rituximab in lupus nephritis, LUNAR, also failed to meet primary endpoints.

\section{Patient population}

Lupus is a very heterogeneous disease and the more targeted our therapies become, the more we will need to recognize subsets of patients who might benefit from any given targeted treatment. In the case of rituximab, disease manifestations caused directly by pathogenic antibodies produced by long-lived plasma cells are unlikely to improve with rituximab since this therapy does not have significant effects on long-lived plasma cells. Based on pre-clinical data, antibody independent mechanisms are important in renal lupus, and short-lived plasma cells may be the predominant source of anti-dsDNA. However, an important caveat to this is the demonstration in murine lupus that anti-dsDNA producing plasma cells can also be selected into the long-lived compartment [28] and may need to be eliminated for optimal efficacy. Open-label studies of B-cell depletion also suggest benefit in refractory disease that has been unresponsive to conventional treatments, including high dose steroids and cyclophosphamide. However, it will not be possible 
to study this in a randomized fashion. These results do raise the additional question as to whether a synergistic effect exists between biologic agents and cyclophosphamide, a point further highlighted by the recently reported failure of LUNAR, where rituximab was combined with mycophenolate mofetil. An ongoing trial of humanized anti-CD20 (ocrelizumab) in lupus nephritis may address this issue given that a subset of patients will receive B-cell depletion therapy combined with cyclophosphamide.

\section{Control regimen}

In both the rituximab and abatacept studies, patients had active disease requiring treatment with moderate to high dose steroids in both the experimental and placebo arms. This is a reasonable design if your experimental therapy has a very strong therapeutic effect, but could miss effects that are still important when tested against an effective treatment. It has been suggested by others that the use of concomitant steroids be restricted as much as possible in future studies and a rapid taper devised [2]. In contrast, the belimumab study did not give a course of steroids at study entry, possibly explaining its relative success - that is, patients were not as sick and there was no course of steroids to obscure the effects of the experimental drug.

\section{Conclusions}

Because of these problems, we believe the door should not be closed on additional studies of B-cell depletion or co-stimulatory blockade in SLE. Based on our current knowledge future studies of biologics could include the following: first, trials with careful attention made to immunological biomarkers of response that allow for treatment modifications if suboptimal effects are seen. In particular, we are concerned about the adequacy of depletion of memory and pathogenic B-cell subsets in SLE after rituximab. The use of biologics needs to be refined and optimized. If B-cell depletion is inadequate in the majority of patients, especially in tissue sites, how can the clinical efficacy of B-cell depletion really be addressed? Second, additional trials of organ specific disease where there may be less heterogeneity and better outcome measures, for example, immune-mediated thrombocytopenia, cutaneous lupus, and central nervous system manifestations of lupus. Third, trials selecting patients based on pathophysiology that might be more responsive to the particular biologic, for example, rituximab treatment may be particularly efficacious for disease manifestations predominantly mediated by B cells and/or short-lived plasma cells (for example, cytopenias) but not those mediated by antibodies produced by long-lived plasma cells. The failure of rituximab in both general and now renal lupus does raise the question of whether reducing pathogenic autoantibodies is key to maximal treatment efficacy. Abatacept may be particularly efficacious for T-cell driven disease manifestations. Of course, in many cases it may be difficult to separate disease subsets cleanly on this basis, and there is mechanistic cross-over (for example, indirect effects of B-cell depletion on T cells). Fourth, long-term trials designed to test whether rituximab or abatacept can prevent flares. Fifth, early intervention trials designed to prevent evolution of the autoimmune response perhaps before selection of autoreactivity into the long-lived plasma cell pool. Lastly, combining rituximab or abatacept with other therapies that might be synergistic, such as low dose cyclophosphamide. Indeed, open-label data in human SLE suggest potential synergy between cyclophosphamide and rituximab, while pre-clinical data in murine lupus nephritis suggest the same for cyclophosphamide and abatacept [20].

In conclusion, we believe that B-cell depletion and co-stimulatory blockade remain promising approaches to the treatment of at least a subset of SLE. Recent randomized clinical trials provide important information regarding optimizing study design and upon further analysis will hopefully yield clues regarding subsets of disease that may be responsive to distinct biologic approaches so that future trials may yield evidencebased proof of efficacy.

\section{Abbreviations}

BAFF, B-cell activating factor of the TNF family; BILAG, British Isles Lupus Assessment Group; CTLA, cytoxic T-lymphocyte antigen; dsDNA, double-stranded DNA; EULAR, European League Against Rheumatism; FDA, Food and Drug Administration; MCR, major clinical response; OMERACT, Outcome Measures in Rheumatology; PCR, partial clinical response; SELENA, Safety of Estrogen in Lupus Erythematosus National Assessment; SLE, systemic lupus erythematosus; SLEDAI, Systemic Lupus Erythematosus Disease Activity Index; SRI, SLE responder index; TNF, tumor necrosis factor.

\section{Competing interests}

JHA has received grants from Amgen Pharmaceuticals and Genentech for mechanistic studies in the context of lupus clinical trials, as well as grants from Proteolix and Vaccinex for drug development in murine models of lupus. She has served as a consultant for Genentech, Roche, Biogen, Proteolix, and Wyeth.

\section{Acknowledgements}

We are grateful to Inaki Sanz and John Looney for intellectual input and stimulating discussions. We thank 
Joan Merrill for reviewing the description of the clinical trial data and providing additional detail.

\section{References}

I. Bongu A, Chang E, Ramsey-Goldman R: Can morbidity and mortality of SLE be improved? Best Pract Res Clin Rheumatol 2002, 16:313-32.

2. Isenberg D, Gordon C, Merrill J, Urowitz M: New therapies in systemic lupus erythematosus - trials, troubles and tribulations - working towards a solution. Lupus 2008, 17:967-70.

3. Dall'Era M, Wofsy D: Clinical trial design in systemic lupus erythematosus. Curr Opin Rheumatol 2006, I 8:476-80.

4. Bertsias G, Gordon C, Boumpas DT: Clinical trials in systemic lupus erythematosus (SLE): lessons from the past as we proceed to the future - the EULAR recommendations for the management of SLE and the use of end-points in clinical trials. Lupus 2008, I 7:437-42.

5. Gordon C, Bertsias GK, loannidis JP, Boletis J, Bombardieri S, Cervera R, Dostál C, Font J, Gilboe IM, Houssiau F, Huizinga TW, Isenberg D, Kallenberg CG, Khamashta MA, Piette JC, Schneider M, Smolen JS, Sturfelt G, Tincani A, Van Vollenhoven R, Boumpas DT: EULAR points to consider in conducting clinical trials in systemic lupus erythematosus. Ann Rheum Dis 2009, 68:470-6.

6. Strand V, Gladman D, Isenberg D, Petri M, Smolen J, Tugwell P: Endpoints: consensus recommendations from OMERACT IV. Outcome measures in rheumatology. Lupus 2000, 9:322-7.

7. US Department of Health and Human Services, Food and Drug Administration, Center for Drug Evaluation and Research (CDER): Guidance for Industry: Systemic Lupus Erythematosus Developing Drugs for Treatment. March 2005. [www.fda.gov/ cder/guidance/6496dft.pdf].

8. Chan OT, Madaio MP, Shlomchik MJ: The central and multiple roles of B cells in lupus pathogenesis. Immunol Rev 1999, I69:|07-2|

9. Shlomchik MJ, Madaio MP, Ni D, Trounstein M, Huszar D: The role of B cells in Ipr/lpr-induced autoimmunity. J Exp Med 1994, I 80: | 295-306.

10. Looney RJ, Anolik JH, Campbell D, Felgar RE, Young F, Arend LJ, Sloand JA, Rosenblatt J, Sanz I: B cell depletion as a novel treatment for systemic lupus erythematosus: A phase I/II dose-escalation trial of rituximab. Arthritis Rheum 2004, 50:2580-9.

II. Isenberg DA: Treating patients with lupus with B-cell depletion. Lupus 2008, I7:400-4.

12. Lindholm C, Borjesson-Asp K, Zendjanchi K, Sundqvist AC, Tarkowski A, Bokarewa M: Longterm clinical and immunological effects of anti-CD20 treatment in patients with refractory systemic lupus erythematosus. J Rheumatol 2008, 35:826-33.

13. Tokunaga M, Saito K, Kawabata D, Imura Y, Fujii T, Nakayamada S, Tsujimura S, Nawata M, Iwata S, Azuma T, Mimori T, Tanaka Y: Efficacy of rituximab (anti-CD20) for refractory systemic lupus erythematosus involving the central nervous system. Ann Rheum Dis 2007, 66:470-5.

FI000 Factor 3.0 Recommended

Evaluated by Gyorgy Nagy 3 I May 2007

14. Podolskaya A, Stadermann M, Pilkington C, Marks SD, Tullus K: B cell depletion therapy for 19 patients with refractory systemic lupus erythematosus. Arch Dis Child 2008, 93:40I-6.

I5. Jónsdóttir T, Gunnarsson I, Risselada A, Henriksson EW, Klareskog L, van Vollenhoven RF: Treatment of refractory SLE with rituximab plus cyclophosphamide: clinical effects, serological changes, and predictors of response. Ann Rheum Dis 2007, 67:330-4.
16. Amoura Z, Mazodier K, Michel M, Viallard J-F, Huong D, Chalumeau N, Cacoub P, Harle JR, Pellegrin JL, Kaplanski G, Godeau B, Piette JC: Efficacy of rituximab in systemic lupus erythematosus: a series of $\mathbf{2 2}$ cases. Arthritis Rheum 2007, 56: S458.

17. Mackay F, Woodcock SA, Lawton P, Ambrose C, Baetscher M, Schneider P, Tschopp J, Browning JL: Mice transgenic for BAFF develop lymphocytic disorders along with autoimmune manifestations. J Exp Med 1999, 190:1697-7I0.

18. Gross J, Johnston J, Mudri S, Enselman R, Dillon SR, Madden K, Xu W, Parrish-Novak J, Foster D, Lofton-Day C, Moore M, Littau A, Grossman A, Haugen H, Foley K, Blumberg H, Harrison K, Kindsvogel W, Clegg $\mathrm{CH}$ : TACI and BCMA are receptors for a TNF homologue implicated in B-cell autoimmune disease. Nature 2000, 404:995-9.

19. Kremer JM, Westhovens R, Leon M, Di Giorgio E, Alten R, Steinfeld S, Russell A, Dougados M, Emery P, Nuamah IF, Williams GR, Becker JC, Hagerty DT, Moreland LW: Treatment of rheumatoid arthritis by selective inhibition of $\mathrm{T}$-cell activation with fusion protein CTLA4Ig. New Engl J Med 2003, 349:1907-I5.

20. Daikh DI, Wofsy D: Cutting edge: reversal of murine lupus nephritis with CTLA4Ig and cyclophosphamide. J Immunol 2001, 166:2913-6.

21. Merrill JT, Neuwelt CM, Wallace DJ, Shanahan JC, Latinis KM, Oates JC, Utset TO, Gordon C, Isenberg DA, Hsieh HJ, Zhang D, Brunetta PG: Efficacy and safety of rituximab in patients with moderately to severely active systemic lupus erythematosus: results from the randomized, double-blind phase II/III study EXPLORER. Presented at 72nd Annual Scientific Meeting of the American College of Rheumatology; October 24-29, 2008; San Francisco, CA. Abstract LI2.

22. Merrill JT, Burgos-Vargas R, Westhovens R, Chalmers A, D'Cruz D, Wallace D, Bae SC, Sigal L, Becker JC, Kelly S, Raghupathi K, Peng Y, Kinaszczuk M, Nash P: The efficacy and safety of abatacept in SLE: results of a 12-month exploratory study. Presented at 72nd Annual Scientific Meeting of the American College of Rheumatology; October 24-29, 2008; San Francisco, CA. Abstract LI5.

23. Chatham W, Furie R, Petri M, Ginzler EM, Wallace DJ, Stohl W, Strand V, Weinstein A, Zhong J, Hough D, Freimuth W: Belimumab (fully human monoclonal antibody to BLyS) improved or stabilized systemic lupus erythematosus (SLE) disease activity over 3 years of therapy. Arthritis Rheum 2008, 58:SI088.

24. Wegener's Granulomatosis Etanercept Trial (WGET) Research Group: Etanercept plus standard therapy for Wegener's granulomatosis. N Engl J Med 2005, 352:35I-6I.

25. Yuen SY, Pope JE: Learning from past mistakes: assessing trial quality, power and eligibility in non-renal systemic lupus erythematosus randomized controlled trials. Rheumatology 2008, 47:I367-72.

26. Anolik JH, Barnard J, Owen T, Zheng B, Kemshetti S, Looney RJ, Sanz I: Delayed memory $B$ cell recovery in peripheral blood and lymphoid tissue in systemic lupus erythematosus after B cell depletion therapy. Arthritis Rheum 2007, 56:3044-56.

27. Anolik JH, Barnard J, Cappione A, Pugh-Bernard AE, Felgar RE, Looney RJ, Sanz I: Rituximab improves peripheral B cell abnormalities in human systemic lupus erythematosus. Arthritis Rheum 2004, 50:3580-90.

28. Hoyer BF, Moser K, Hauser AE, Peddinghaus A, Voigt C, Eilat D, Radbruch A, Hiepe F, Manz RA: Short-lived plasmablasts and long-lived plasma cells contribute to chronic humoral autoimmunity in NZB/W mice. J Exp Med 2004, I99: I577-84.

FI000 Factor 3.0 Recommended Evaluated by Ken Smith 17 Jun 2004 